\title{
Modeling of light-sensitive resonant-tunneling-diode devices
}

\author{
I. J. S. Coêlho and J. F. Martins-Filhoa) \\ Grupo de Fotônica, Departamento de Eletrônica e Sistemas, Universidade Federal de Pernambuco, \\ 50740-530 Recife-PE, Brazil
}

\section{J. M. L. Figueiredo}

Departamento de Fisica da Faculdade de Ciências e Tecnologia da Universidade do Algarve, 8000-062 Faro, Portugal

\author{
C. N. Ironside \\ Department of Electronics and Electrical Engineering, University of Glasgow, Glasgow G12 8LT, \\ United Kingdom
}

(Received 5 November 2003; accepted 9 March 2004)

\begin{abstract}
We present a method to include the effects of light excitation on two different models of resonant-tunneling-diode-based devices. Our approach takes into account both photoconductive and charge accumulation effects responsible for shifting the static $I-V$ curve when the structure is under light excitation. Computational simulations led to good agreement between the model and experimental results. (C) 2004 American Institute of Physics. [DOI: 10.1063/1.1728290]
\end{abstract}

\section{INTRODUCTION}

The resonant tunneling diode (RTD) attracts interest due to its highly nonlinear static current-voltage characteristic, ${ }^{1}$ which exhibits a range with negative differential resistance. The recent interest in applying RTDs for optical modulation, ${ }^{2}$ switching $^{3-5}$ and photodetection ${ }^{6-8}$ has demanded efficient models to explain their behavior under light excitation. The work of Moise et al. ${ }^{6}$ has shown that the RTD integrated with a photodetector is a highly sensitive device (10 A/W). For high-speed optical communication systems, the RTD integrated with a photodetector could provide an integrated solution to the currently employed, hybrid arrangement of a separate photodector and transimpedance amplifier. However, at this stage in its development, the RTD photodetector (RTD-PD) needs to be modeled; such modeling should be able to give insight to RTD physics and, by means of widely available computer-aided design tools, to predict accurately its behavior under various operating conditions.

The models proposed by Brown et al. ${ }^{9}$ and Schulman et al. ${ }^{10}$ relate flow of carriers through the double-barrier structure (DBS) to the bias conditions set across the RTD. They considered coherent and noncoherent current flowing components. Their models are Simulation Program with Integrated Circuits Emphasis (SPICE) compatible and suitable for simulation under dark conditions only. Our purpose is to extend the physics-based model of Schulman et al. and the SPICE model of Brown et al. to include the effect of illumination and to validate our model by comparing it with existing data on the change of the dc current voltage curve under light excitation.

In this article, we describe a general method to include light-induced effects in physics-based models of optoelectronic RTDs such as RTD-PD. We take into account the photoconductive and charge accumulation effects close to the

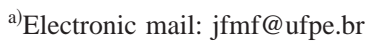

DBS. These two effects have been referred to as responsible for the typical experimentally observed light-induced shifting of RTD dc current-voltage characteristic. ${ }^{3,4}$ We calculate the contribution of the two effects and predict the device behavior under light excitation from the radiation characteristics and device parameters. Computational simulations were performed aiming to compare theoretical results from the proposed model to experimental data found in the literature. $^{5}$

\section{MODELING}

We propose an improvement in the original models developed by Brown et al. and Schulman et al. through the introduction of two terms to account for the effect of light. The original dependence of current on voltage is maintained for dark conditions. Therefore, following the formulation of Brown et al., ${ }^{9}$ we propose

$$
\begin{aligned}
I= & C_{1}\left(V+R_{\mathrm{eq}} I+V_{\mathrm{ph}}\right)^{3}\left\{\tan ^{-1}\left[C_{2}\left(V+R_{\mathrm{eq}} I+V_{\mathrm{ph}}-V_{T}\right)\right]\right. \\
& \left.-\tan ^{-1}\left[C_{2}\left(V+R_{\mathrm{eq}} I+V_{\mathrm{ph}}-V_{N}\right)\right]\right\} \\
& +C_{3}\left(V+R_{\mathrm{eq}} I+V_{\mathrm{ph}}\right)^{5}+C_{4}\left(V+R_{\mathrm{eq}} I+V_{\mathrm{ph}}\right)^{3}
\end{aligned}
$$

and Schulman et al., ${ }^{10}$

$$
\begin{aligned}
I= & A \ln \left\{\frac{1+e^{e\left[B-C+n_{1}\left(V+R_{\mathrm{eq}} I+V_{\mathrm{ph}}\right) / k T\right]}}{\left.1+e^{e\left[B-C-n_{1}\left(V+R_{\mathrm{eq}} I+V_{\mathrm{ph}}\right) / k T\right]}\right\}}\right. \\
& \times\left\{\frac{\pi}{2}+\tan ^{-1}\left[\frac{C-n_{1}\left(V+R_{\mathrm{eq}} I+V_{\mathrm{ph}}\right)}{D}\right]\right\} \\
& +H\left[e^{n_{2} e\left(V+R_{\mathrm{eq}} I+V_{\mathrm{ph}}\right) / k T}-1\right],
\end{aligned}
$$

where $V_{\mathrm{ph}}$ is a photoinduced voltage (dependent on the light intensity shone on the structure) due to the charge accumulation effect, and $R_{\text {eq }}$ is an equivalent series resistance which accounts for the photoconductive effect and/or for the (fixed) resistances of the bias circuitry. The calculation of these two terms will be discussed in Sec. III. The other factors are 


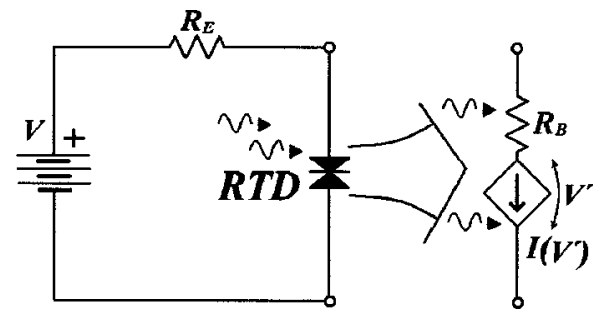

FIG. 1. Biasing circuit and the voltage-controlled current source equivalent model of the RTD-PD. $R_{E}$ is the external resistance attributed to the contacts, wires and/or bias circuitry; $R_{B}$ is the internal (built-in) resistance related to the layers surrounding the DBS.

fitting parameters - as originally defined by the authors, $C_{1}$, $C_{2}, C_{3}$ and $C_{4}$ (see Ref. 9) in Eq. (1) and $A, B, C, D, H, n_{1}$ and $n_{2}$ (see Ref. 10) in Eq. (2)—obtained from the currentvoltage curve measured under dark conditions. Therefore, the effect of light is taken into account by the $V_{\text {ph }}$ and $R_{\text {eq }}$ terms only.

A first interpretation for Eqs. (1) and (2) is that they represent nonlinear voltage-controlled current sources that describe the RTD dc behavior, and are dependent on the intensity of light as illustrated in Fig. 1. Impinging photons modulate both structural layers' resistivity and accumulated charge populations in the spacer layer (collector side) of the RTD.

\section{ESTIMATION OF PARAMETERS}

\section{A. Series resistances}

Under dark conditions, the series resistance $\left(R_{S}\right)$ associated to the layers placed between the electrodes and the DBS and due to the bias circuitry is given by

$$
R_{S}=R_{B}+R_{E},
$$

where $R_{B}$ is the internal resistance due to the layers beside the DBS and $R_{E}$ is an external resistance associated to the contacts, wires and other bias circuit components (Fig. 1 depicts the suggested dc equivalent electronic modeling). Such a resistance $R_{S}$ would be responsible for a drop in voltage from the power supply $V$ to the voltage effectively applied across the DBS $\left(V^{\prime}\right)$. This effective applied voltage $\left(V^{\prime}\right)$ drives the current $I$ in a similar way, as empirically suggested in the Refs. 9 and 10.

Under dark conditions, one expects

$$
V^{\prime}=V-R_{S} I
$$

to control tunneling probability through the DBS. On the other hand, absorption of impinging photons in the semiconductor layers surrounding the DBS leads to the variation of their resistivity. This variation can be expressed analytically as

$$
\Delta \rho=\frac{-\Delta \sigma}{\sigma(\sigma+\Delta \sigma)},
$$

where $\Delta \sigma$ is the increase in conductivity due to the photoinduced hole-and-electron populations $\delta p=\delta n$. Equation (5) is obtained assuming $\rho+\Delta \rho=1 /(\sigma+\Delta \sigma)$ for large values of $\Delta \sigma$, that is, for $\Delta \sigma$ on the same order of $\sigma$. For the case in which $\Delta \sigma$ is much smaller than $\sigma$, Eq. (5) is reduced to $\Delta \rho$ $=-\Delta \sigma / \sigma^{2}$. However, this is not the case for moderate levels of illumination. Simple calculations can be performed to verify this condition.

The decrease in resistivity given by Eq. (5) leads to the change in the built-in resistance $R_{B}$, given by

$$
\Delta R_{B}=\Delta \rho \frac{l}{A}-(\rho+\Delta \rho) \frac{x_{a}}{A},
$$

which turns Eq. (4) into

$$
V^{\prime}=V-\left(R_{S}+\Delta R_{B}\right) I
$$

In Eq. (6), $l$ and $A$ are the total thickness and sectional area of the surrounding layers, respectively, and $x_{a}$ is the hole accumulation region length (adjacent to the DBS), which effectively reduces the structural layer thickness from its original length $l$ to $l-x_{a}$.

Re-expressing Eq. (5) in terms of the equilibrium populations $p_{0}$ and $n_{0}$, their respective mobilities in the material $\mu_{e}$ and $\mu_{h}$, and the photoinduced carrier density $\delta p$, we get

$$
\Delta \rho=\frac{-\left(\mu_{e}+\mu_{h}\right) \delta p}{e\left(\mu_{e} n_{0}+\mu_{h} p_{0}\right)\left[\left(\mu_{e} n_{0}+\mu_{h} p_{0}\right)+\left(\mu_{e}+\mu_{h}\right) \delta p\right]} .
$$

An interesting aspect related to this expression arises for high levels of photogeneration in lightly doped spacer layers. In this case, Eq. (8) revels the saturation of the decrease in resistivity on the optical laser intensity. More than this, $\Delta \rho$ tends numerically to compensate the effect of the built-in resistance when $\left(\mu_{e} n_{0}+\mu_{h} p_{0}\right) \ll\left(\mu_{e}+\mu_{h}\right) \delta p$ since Eq. (8) becomes [see Eqs. (6) and (7)]

$$
\Delta \rho \approx-\rho=\frac{-1}{e\left(\mu_{e} n_{0}+\mu_{h} p_{0}\right)} .
$$

In other words, Eq. (9) is meaningful only at high optical powers when almost all of the applied voltage appears across the DBS and drives the tunneling probabilities, so that Eq. (7) would become

$$
V^{\prime} \cong V-R_{E} I
$$

since we did not consider (up to this point) the electrostatic field arising due to the effect of the hole accumulation in the collector side of the DBS. Notice that it does not matter how thick $x_{a}$ is in Eq. (6), $\Delta R_{B}$ will always cancel $R_{B}$ when the optical powers are high enough. In other words, high levels of illumination make layers behave more conductively so that a negligible drop in voltage is expected across them.

It should be clear that under dark conditions, $R_{B}$ distorts the dc characteristic of RTD-PD devices due to the voltage drop, as given by Eqs. (3) and (4). Therefore, the fitting parameters of Eqs. (1) and (2) evaluated under dark condition are affected by this distortion. When illuminated, the resistivity decreases, canceling the effect of $R_{B}$, as given by Eqs. (6)-(9). Therefore, it is clear that $R_{\text {eq }}=\Delta R_{B}$ in our model, and it is obtained from Eqs. (6), (8), and (9). 


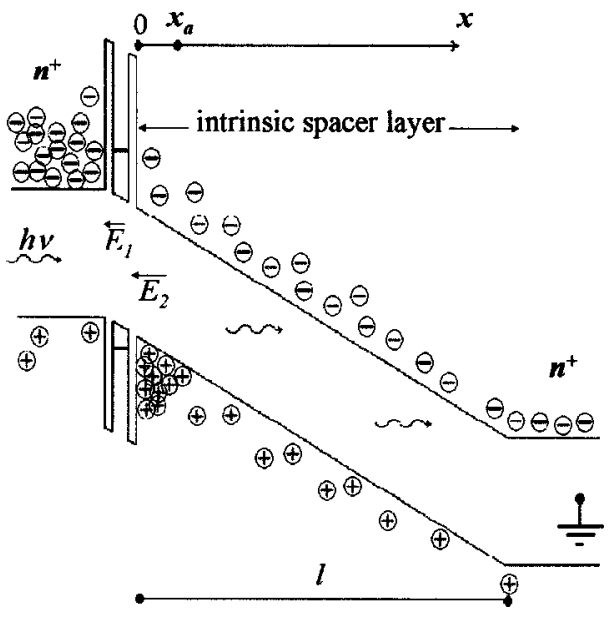

FIG. 2. Schematic diagram of the typical RTD-PD band structure (we show the bottom of the conduction band and top of the valence band as a function of distance) and description of the absorption, accumulation, and tunneling processes. Black circles represent thermally generated carriers, while gray circles represent photogenerated carriers.

\section{B. Photoinduced voltages}

We characterize the effect of light on the currentvoltage curve as an additional photoinduced drop in voltage across the DBS. The model we use to estimate the photoinduced voltage assumes the typical RTD-PD structure, as illustrated in Fig. 2. Typical devices for photodetection purposes employ a DBS with thicker lower-doped collectorspacer layers. ${ }^{5,6}$ Their overall behavior results from modulation of the carrier's population by the impinging photons (outer layers are made much more conductive for electrode-matching purposes).

As carriers are photogenerated within the surrounding areas of the DBS, excess holes accumulate beside the right side of the double barrier (see Fig. 2), in the collector-spacer layer. This effect is balanced by recombination and tunneling phenomena, leading to a steady excess population $p$ close to the DBS. Such a charge distribution is responsible for two internal electrostatic fields, labeled $E_{1}$ and $E_{2}$. As suggested earlier, ${ }^{9,10}$ the voltage across the DBS drives the current because it sets tunneling probability for electrons. Additional voltages, which are expected to exist there, are directly related to $E_{1}$ and $E_{2}$.

Photogenerated charges are swept out of the thicker layer towards its boundaries due to the external biasing (see Fig. 2). Holes in the valence band are assumed to pile up close to the DBS uniformly spread over the interface, while photogenerated electrons are removed by the applied voltage towards the collector contact. Equilibrium in the population of holes for a certain bias voltage is sustained by a balance between arrival rate of photogenerated holes, and recombination rate in the accumulation layer. There is a charge distribution per unit area $\rho_{S}\left(\mathrm{C} / \mathrm{cm}^{2}\right)$ in a sheet whose thickness is negligible when compared to the transversal dimensions. Such a density of charges is related to the electrostatic field across the barrier layer by

$$
D_{1 b}=\varepsilon_{b} E_{1 b}=\rho_{S},
$$

where the subscript " 1 " reminds us that this is part of the additional fields mentioned earlier.

At the interfaces between layers implementing barriers and well there are no other sheets of static charges, so that the usual boundary condition guarantees

$$
D_{1 w}=\varepsilon_{w} E_{1 w}=\varepsilon_{b} E_{1 b}=D_{1 b} .
$$

Equations (11) and (12) allow us to estimate the total drop in voltage through the DBS due to the accumulation of holes (if $\rho_{S}$ are known) by

$$
V_{1}=\frac{2 \varepsilon_{w} l_{b}+\varepsilon_{b} l_{w}}{\varepsilon_{w} \varepsilon_{b}} \rho_{S}
$$

where $l_{w}$ and $l_{b}$ are the thicknesses of the layers of the DBS, and $\varepsilon_{w}$ and $\varepsilon_{b}$ are their respective permittivities. Therefore, $V_{\text {ph }}$ in Eqs. (1) and (2) is the $V_{1}$ given by Eq. (13), and it is due to $E_{1}$, as illustrated in Fig. 2. It can be evaluated for each optical power, upon the estimation of $\rho_{S}$.

To estimate $\rho_{S}$, one could consider the thickness of that sheet composed of accumulated holes to set up an $x$-dependent electrostatic field (say $E_{2}$ ) through the extent $x_{a}$ (see Fig. 2). For sake of simplicity, the charge is supposed to be uniformly distributed along the thickness of this region whose volume is given by $x_{a} A$, and whose hole concentration is referred to as $p$. That means the total accumulated charge could be expressed by

$$
Q_{a}=e p x_{a} A,
$$

where $e$ is the elementary charge. In addition, the electrostatic field

$$
\vec{E}_{2}(x)=\frac{-e\left(x_{a}-x\right) p}{\varepsilon_{i}} \hat{a}_{x}
$$

is integrated from $x=0$ to $x=x_{a}$, giving the drop in voltage through the hole accumulation layer $V_{2}$ (due to $E_{2}$ ) in Fig. 2, as

$$
V_{2}=\frac{e x_{a}^{2} p}{2 \varepsilon_{i}} .
$$

In addition, from standard theory for semiconducting materials, ${ }^{11}$ the quasi-Fermi level shift for holes inside and outside of the hole accumulation layer allows one to write, according to the Joyce-Dixon approximation, ${ }^{12}$

$$
V_{2} \cong \frac{k T}{e}\left[\ln \left(\frac{p}{\delta p}\right)+\frac{p-\delta p}{\sqrt{8} N_{v}}\right],
$$

where $N_{v}$ is the density of states at the top of the valence band for that layer.

Equating (16) to (17) leads to an important relation between $p$ and $x_{a}$ for a given bias voltage. Searching for a desirable second equation relating $p$ to $x_{a}$, it seems to be sensible, expressing a simple rate equation that neglects tunneling holes as well as photogeneration taking place in the accumulation layer. While excess holes are being dragged to the accumulation layer from beyond $x_{a}$ (by the applied field), the rate at which they arrive can be written as 


$$
A_{R}=\frac{v_{h} \delta p}{x_{a}}
$$

where $v_{h}$ is the drift velocity for holes. Accumulated holes recombine at a rate given by

$$
D_{R} \cong \frac{p}{\tau_{0}},
$$

where $\tau_{0}$ is the lifetime of electron-hole pairs. We assume that for each bias voltage, $A_{R}$ is equal to $D_{R}$, which give us the other desired equation relating $p$ to $x_{a}$.

Determining the photoinduced voltage $V_{\mathrm{ph}}=V_{1}$ in Eqs. (1) and (2), for given conditions of illumination and biasing, depends on the task of solving the nonlinear system for $p$ and $x_{a}$, given by

$$
\left\{\begin{array}{l}
p x_{a}^{2}=\frac{2 \varepsilon_{i} k T}{e^{2}}\left[\ln \left(\frac{p}{\delta p}\right)+\frac{p-\delta p}{\sqrt{8} N_{v}}\right] \\
p x_{a} \cong v_{h} \tau_{0} \delta p
\end{array}\right.
$$

since the calculation of $p$ and $x_{a}$ leads to the calculation of $\rho_{S}$, and therefore $V_{1}$ is obtained by means of Eq. (13).

In summary, our model has two major components. There is a shift in the current-voltage curve to lower voltage (i.e., the whole curve moves to the left) due to the reduced resistance of the epilayers surrounding the DBS caused by photoinduced carriers. This is the same effect discussed in a qualitative way by Moise et al. ${ }^{6}$ but here we have a quantitative model based on the entirely electrical models of Brown et al. ${ }^{9}$ and Schulman et al. ${ }^{10}$ We have also included the effect of fields induced at the accumulation layer by photoinduced holes. With this model, we are able to obtain quantitative predictions of the RTD-PD behavior.

\section{SIMULATIONS AND RESULTS}

Here, we use the formulations presented in Secs. II and III to model the effect of light on the dc current-voltage curve of the RTD-PD. Different RTD structures have been reported, and discussions about which is the governing process that leads to shifting the current-voltage curve are profuse. ${ }^{5,6,13,14}$ We have chosen a particular structure in the literature for analysis, aiming to obtain simulation results for discussion. ${ }^{5}$ The choice was based on three reasons: (1) all features of the laser radiation as well as the structural parameters of the RTD-PD were reported in Ref. 5; (2) the thicker collector-spacer layer besides the DBS is intrinsic, which is particularly suitable for our formulations; and (3) experimental $I-V$ curves, showing shifting for different levels of optical power, are available, allowing comparisons to the expected performance based on the models we have adopted and handled. Reported $I-V$ curves in the darkness and under laser irradiation became available for analysis by picking the points up from figures of Ref. 5. The dark curve was assumed to express the "intrinsic curve," that is, it shows the actual behavior of the RTD regardless any possible series resistances. Thus, the digitized data are fitted in Fig. 3 for initial estimation of the parameters of Eqs. (1) and (2) under dark conditions. We removed the data in the region between the peak and the valley (the NDR region). Since the points in

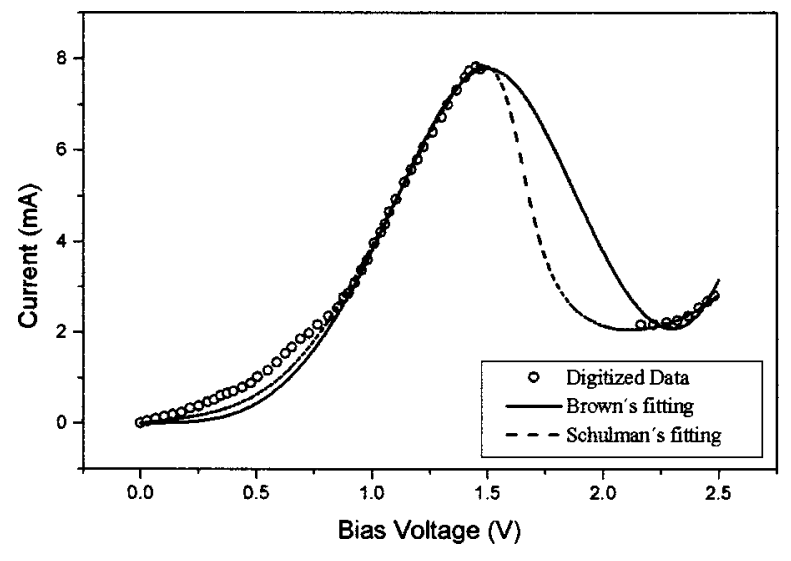

FIG. 3. Obtained fitting to picked data under dark conditions. This is the assumed current-voltage curve of the device (no external resistances are assumed to be connected to or built-in the RTD). Circles: digitized experimental data (see Ref. 5); line: obtained fitting based on the Brown's modeling $\left(C_{1}=3.44157 \times 10^{-3}, C_{2}=1.87204, C_{3}=3.51557 \times 10^{-4}\right.$, and $C_{4}$ $=-3.03362 \times 10^{-3}$ are the fitting parameters). Dash: obtained fitting based on the Schulman's modeling $\left(A=7.46 \times 10^{-4}, B=0.130, C=0.2305, D\right.$ $=0.017, H=2.4 \times 10^{-6}, n_{1}=0.141$, and $n_{2}=0.069$ are the fitting parameters).

this region were measured when the device was oscillating, they should not be considered for the dc response of the device. Therefore, our model does not take into account selfoscillation.

Next, with the fitting parameters already available, we can evaluate the effects of the laser light by estimating the voltage terms $R_{\text {eq }} I$ and $V_{\text {ph }}$ to Eqs. (1) and/or (2).

For experimental conditions described in Ref. 5 where the intrinsic semiconducting layer exhibits $N_{v}=6.4$ $\times 10^{18} \mathrm{~cm}^{-3}$, for the wavelength $\lambda_{0}=860 \mathrm{~nm}$, the approximate value for intrinsic absorption ${ }^{12}$ is $\alpha(\operatorname{InGaAs}) \sim 2$ $\times 10^{4} \mathrm{~cm}^{-1}$. We neglected optical losses due to reflections and/or absorption by free carriers in the heavily doped shallower layers. The estimated generation rate $^{12}$ at $P_{\text {opt }}$ $=2 \mathrm{~mW}$ is $G \sim 6 \times 10^{24} \mathrm{~cm}^{-3} \mathrm{~s}^{-1}$. Thus, the exceeding photogenerated population for that power is estimated to be $\delta p$ $=6.5 \times 10^{15} \mathrm{~cm}^{-3}\left(\tau_{0}=1.06 \times 10^{-9} \mathrm{~s}\right)$. Therefore, Eq. (20) gives us the steady-state volumetric concentration of holes $p=3.3 \times 10^{17} \mathrm{~cm}^{-3}$, and the thickness of the accumulation region $x_{a}=21 \mathrm{~nm}$. Taking these values for $p$ and $x_{a}$ in Eq. (14), it is possible to estimate $\rho_{S}=Q_{a} / A \sim 1.1$ $\times 10^{-7} \mathrm{C} \mathrm{cm}^{-2}$. From Eq. (13), one can obtain $V_{1}=V_{\mathrm{ph}}$ $=119 \mathrm{mV}$, for $P_{\mathrm{opt}}=2 \mathrm{~mW} .{ }^{5} \mathrm{It}$ is then possible to obtain the key parameter in our model, $V_{1}=V_{\mathrm{ph}}$, for any optical power level. In other words, solving system (20) allows one to estimate, for each optical power of the laser beam, the additional voltage drop across the DBS (due to the accumulation of holes besides the inner barrier), which increases the tunneling probability for electrons, left-shifting the dc characteristic of the RTD by a predetermined amount.

Accounting for the conductivity modulation of the intrinsic layer, we assumed that there is a steady-state concentration of traveling electrons $n_{0} \gg p_{0}$ under dark conditions in that material, due to tunneling/crossing-over the DBS at high enough bias voltages. Near the peak current $\left(I_{p}=7.7 \mathrm{~mA}\right){ }^{5}$ $n_{0}=3.4 \times 10^{14} \mathrm{~cm}^{-3} \gg n_{i}=1.2 \times 10^{11} \mathrm{~cm}^{-3}$. From Eqs. (6)- 


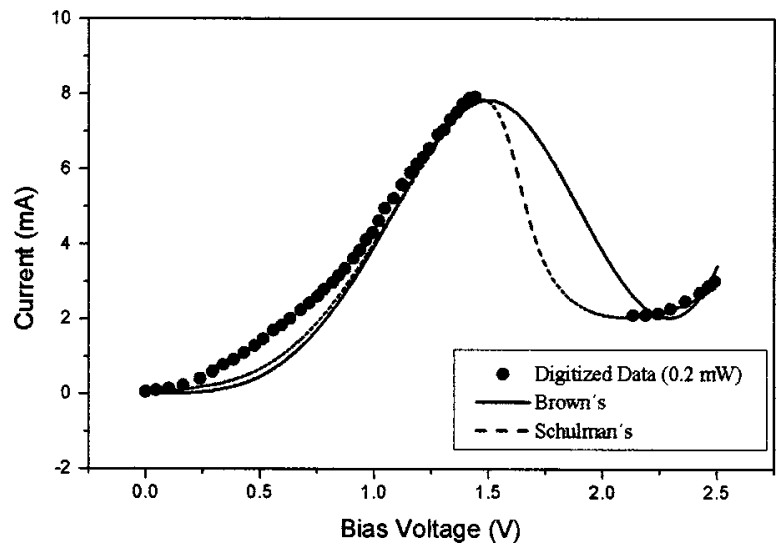

FIG. 4. Experimental and modeled curves for RTD under illumination $\left(P_{\mathrm{op}}=0.2 \mathrm{~mW}\right)$. Circles: digitized experimental data (see Ref. 5). Line: obtained curve based on the modified Brown's modeling (same values for $C_{1}$, $C_{2}, C_{3}$, and $C_{4}$ as in dark conditions). Dash: obtained curve based on the modified Schulman's modeling (same values for $A, B, C, D, H, n_{1}$, and $n_{2}$ as in the dark). Calculated parameters for $P_{\mathrm{op}}=0.2 \mathrm{~mW}: V_{\mathrm{ph}}=10,5 \mathrm{mV}$, and $R_{\mathrm{eq}} I=4.5 \mathrm{mV}$.

(9), we concluded that in the limit of high optical power, $\Delta \rho \rightarrow-\rho$ (meaning that there is a reduction in the resistivity level of the semiconductor layer by an amount that is equal to the resistivity value) when $\delta p \gg n_{0}\left(P_{\text {opt }} \gg 0.1 \mathrm{~mW}\right)$. Resistivity will depend on the current level, however, showing its higher value in the valley $(7.5 \Omega \mathrm{cm})$ and lower value in the peak $(1.7 \Omega \mathrm{cm})$. The corresponding intrinsic resistance values are estimated as $R_{B v}=2.7 \Omega$ and $R_{B p}=0.6 \Omega$, respectively, leading to almost the same value for the ohmic drop in voltage $R_{B v} I_{v}=R_{B p} I_{p}=4.5 \mathrm{mV}$. This is the maximum shift of the dc characteristic of the device due only to the photoconductivity effect, when the device is illuminated. This contribution is much smaller than the hole accumulation effect estimated earlier, which is $V_{1}=V_{\mathrm{ph}}=119 \mathrm{mV}$, for $P_{\mathrm{opt}}$ $=2 \mathrm{~mW}$, for instance.

Theoretical curves were plotted from Eqs. (1) and (2), along with experimental data from Ref. 5 for different levels

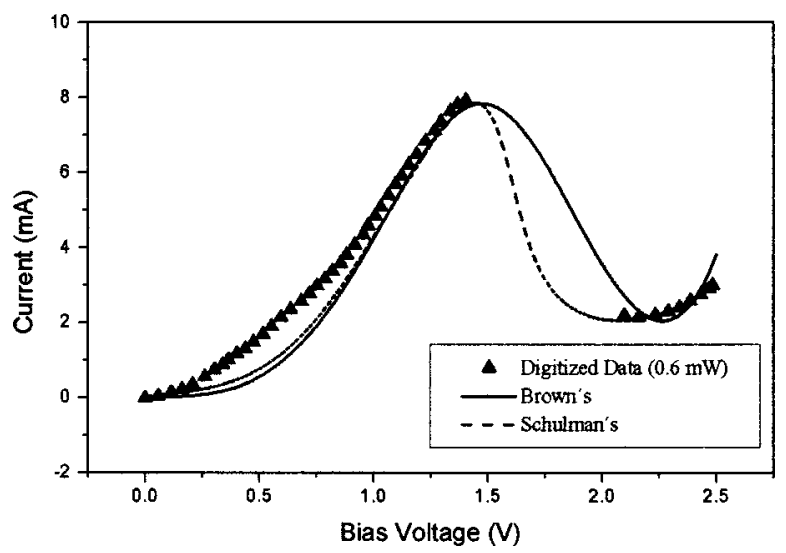

FIG. 5. Experimental and modeled curves for RTD under illumination $\left(P_{\mathrm{op}}=0.6 \mathrm{~mW}\right)$. Up-triangles: digitized experimental data (see Ref. 5). Line: obtained curve based on the modified Brown's modeling (same $C_{1}$, $C_{2}, C_{3}$, and $C_{4}$ as in dark). Dash: obtained curve based on the modified Schulman's modeling (same $A, B, C, D, H, n_{1}$, and $n_{2}$ as in dark). Calculated parameters for $P_{\mathrm{op}}=0.6 \mathrm{~mW}: V_{\mathrm{ph}}=36 \mathrm{mV}$, and $R_{\mathrm{eq}} I=4.5 \mathrm{mV}$.

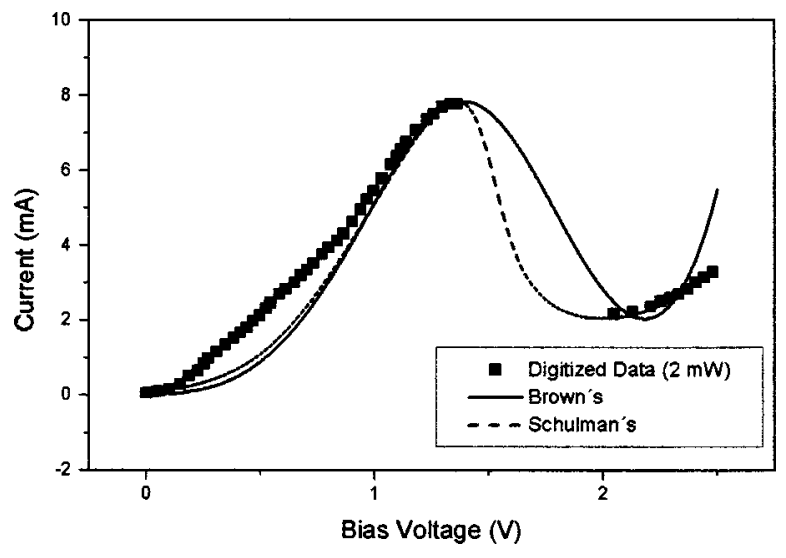

FIG. 6. Experimental and modeled curves for RTD under illumination $\left(P_{\mathrm{op}}=2 \mathrm{~mW}\right)$. Squares: digitized experimental data (see Ref. 5). Line: obtained curve based on the modified Brown's modeling (same $C_{1}, C_{2}, C_{3}$, and $C_{4}$ as in dark conditions). Dash: obtained curve based on the modified Schulman's modeling (same $A, B, C, D, H, n_{1}$, and $n_{2}$ as in dark). Calculated parameters for $P_{\mathrm{op}}=2 \mathrm{~mW}: V_{\mathrm{ph}}=119 \mathrm{mV}$, and $R_{\mathrm{eq}} I=4.5 \mathrm{mV}$.

of illumination in Figs. 4-7. The calculated values for $V_{\mathrm{ph}}$ and $R_{\text {eq }}$ for each optical power level are: $V_{\mathrm{ph}}=10.5 \mathrm{mV}$ and $R_{\mathrm{eq}} I=4.5 \mathrm{mV}$ for $P_{\mathrm{op}}=0.2 \mathrm{~mW}, V_{\mathrm{ph}}=36 \mathrm{mV}$ and $R_{\mathrm{eq}} I$ $=4.5 \mathrm{mV}$ for $P_{\mathrm{op}}=0.6 \mathrm{~mW}, V_{\mathrm{ph}}=119 \mathrm{mV}$ and $R_{\mathrm{eq}} I$ $=4.5 \mathrm{mV}$ for $P_{\mathrm{op}}=2 \mathrm{~mW}, V_{\mathrm{ph}}=290 \mathrm{mV}$ and $R_{\mathrm{eq}} I=4.5 \mathrm{mV}$ for $P_{\mathrm{op}}=5 \mathrm{~mW}$. The modeled curves show good agreement with the experimental ones in Figs. 4 to 6. In Fig. 7, we observe the agreement between theoretical and experimental curves is poorer. This can be due to tunneling of holes through the DBS, which causes a reduction of the hole concentration in the accumulation region. Since our model does not take this effect into account, the theoretical curves overestimate the $V_{\mathrm{ph}}$ for high optical powers.

From Figs. 4 to 7, we can conclude that the theoretical curves obtained from the Schulman-based model give a better match to the experimental results than the ones obtained from the Brown-based model. Although the original models are SPICE compatible, ${ }^{9,10,15,16}$ we implemented them in

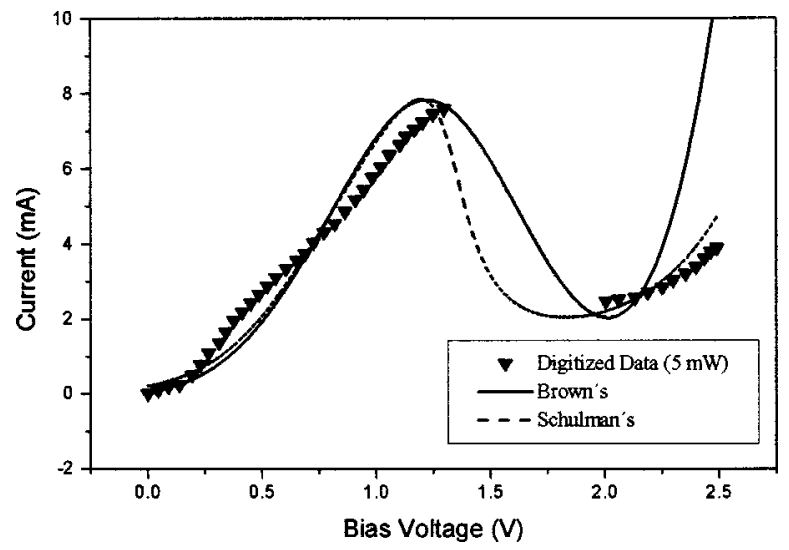

FIG. 7. Experimental and modeled curves for RTD under illumination $\left(P_{\text {op }}=5 \mathrm{~mW}\right)$. Down-triangles: digitized experimental data (see Ref. 5). Line: obtained curve based on the modified Brown's modeling (same $C_{1}$, $C_{2}, C_{3}$, and $C_{4}$ as in dark). Dash: obtained curve based on the modified Schulman's modeling (same $A, B, C, D, H, n_{1}$, and $n_{2}$ as in dark). Calculated parameters for $P_{\mathrm{op}}=5 \mathrm{~mW}: V_{\mathrm{ph}}=290 \mathrm{mV}$, and $R_{\mathrm{eq}} I=4.5 \mathrm{mV}$. 
MATHCAD ${ }^{\mathrm{TM}}$, using a differential correction method and the successive approximations method to obtain the fitting parameters for the dark condition for the Brown's and Schulman's models, respectively.

\section{CONCLUSIONS}

We presented a general method to include the effect of light on two static models for dc current-voltage characteristics of resonant-tunneling-diode-based devices. Our method takes into account the two physical processes usually discussed in the literature: photoconductivity and charge accumulation effects in double-barrier RTD structures. Our model uses the same fitting parameters for the device $I-V$ curve in dark conditions and then simulates and predicts the device's behavior under an illumination condition. We performed computer simulations of our model using MATH$\mathrm{CAD}^{\mathrm{TM}}$ with parameter estimations calculated from experimental data of previously published results. The currentvoltage curves obtained with our model have matched the experimental data found in the literature, and also have given insights into the role of each physical process taking place in that particular device. For the RTD-PD device analyzed here, we found that the photoconductivity effect is negligible compared to the charge accumulation effect for optical powers above $0.6 \mathrm{~mW}$.

We neglected tunneling of holes to keep the same formalisms developed earlier in the assumed physics-based models for RTDs. Very often, only tunneling conditions for electrons are considered formally for $I-V$ curves under darkness, and we kept the same assumption here. It is crucial to set accumulation conditions by the DBS sides, where the depletion of holes is assumed to be via recombination with tunneling electrons only. However, it is sensible to presume that this latter hypothesis weakens for higher optical powers. This may explain the poor agreement between our prediction and the actual device behavior for the highest laser intensity case analyzed.

Similar to previously reported modeling work, our model is based on an equivalent circuit, and it also contains adjustable parameters. However, these parameters are obtained in a systematic way from the dark current-voltage curves and from physics-based simple equations. We believe it can be a useful tool for RTD based device design and circuit simulations for applications in optical switching and photodetection. Future work will include adapting the model to simulate the high-frequency characteristics of RTD-PDs.

\section{ACKNOWLEDGMENTS}

The authors would like to thank Eric Arantes and Sergio Campello, from the Photonics Group-DES-UFPE, for their help with computer programming and simulations. One of the authors (I. J. S. C.) acknowledges scholarship support from the Brazilian agency $\mathrm{CNPq}$.

${ }^{1}$ J. P. Sun, G. I. Haddad, P. Mazumder, and J. N. Schulman, Proc. IEEE 86, 641 (1998).

${ }^{2}$ J. M. L. Figueiredo, A. R. Boyd, C. R. Stanley, C. N. Ironside, S. G. McMeekin, and A. M. P. Leite, Appl. Phys. Lett. 75, 3443 (1999).

${ }^{3}$ P. England, J. E. Golub, L. T. Florez, and J. P. Harbison, Appl. Phys. Lett. 58, 887 (1991).

${ }^{4}$ P. W. Park, H. Y. Chu, S. G. Han, Y. W. Choi, G. Kim, and El-Hang Lee, Appl. Phys. Lett. 67, 1241 (1995).

${ }^{5}$ T. S. Moise, Y.-C. Kao, L. D. Garret, and J. C. Campbell, Appl. Phys. Lett. 66, 1104 (1995)

${ }^{6}$ T. S. Moise, Y.-C. Kao, C. L. Goldsmith, C. L. Schow, and J. C. Campbell, IEEE Photonics Technol. Lett. 9, 803 (1997).

${ }^{7}$ J. F. Martins-Filho, R. E. de Araujo, A. S. L. Gomes, J. M. L. Figueiredo, C. R. Stanley, and C. N. Ironside, in Proc. IEEE LEOS 2000, 13th Annual Meeting, Rio Grande, Puerto Rico, 2000, p. 15-16.

${ }^{8}$ J. F. Martins-Filho, R. E. de Araujo, A. S. L. Gomes, J. R. Rios Leite, J. M. L. Figueiredo, C. R. Stanley, and C. N. Ironside, in Tech. Dig. SBMO/ IEEE MTT-S IMOC 2001-International Microwave and Optoelectronics Conference, Belém, PA, Brazil, 2001, p. 325-328.

${ }^{9}$ E. R. Brown, O. B. McMahon, L. J. Mahoney, and K. M. Molvar, Electron. Lett. 32, 938 (1996).

${ }^{10}$ J. N. Schulman, H. J. De Los Santos, and D. H. Chow, IEEE Electron Device Lett. 17, 220 (1996).

${ }^{11}$ R. S. Muller and T. I. Kamins, Device Electronics for Integrated Circuits, 2nd ed. (Wiley, New York, 1985).

${ }^{12} \mathrm{~J}$. Singh, Semiconductor Optoelectronics, Physics and Technology (McGraw-Hill, New York, 1995), p. 238ff.

${ }^{13}$ S. C. Kan, S. Wu, S. Sanders, G. Griffel, and A. Yariv, J. Appl. Phys. 69, 3384 (1991).

${ }^{14}$ H. S. Li, L. P. Chen, Y. W. Chen, K. L. Wang, D. S. Pan, and J. M. Liu, Appl. Phys. Lett. 65, 2999 (1994).

${ }^{15}$ M. Bhattacharya and P. Mazumder, IEEE Trans. Comput.-Aided Des. 20, 39 (2001).

${ }^{16}$ T. P. E. Broekaert, B. Brar, J. P. A. Van der Wagt, A. C. Seabaugh, F. J. Morris, T. S. Moise, E. A. Beam, and G. A. Frazier, IEEE J. Solid-State Circuits 33, 1342 (1998). 